\title{
La contabilidad de costos y rentabilidad en la Pyme
}

\author{
Domingo García Pérez de Lema* \\ Salvador Marín Hernández** \\ Francisco Javier Martínez García***
}

\section{Resumen}

Este trabajo tiene dos objetivos: por un lado, analizar las características de las empresas que tienen implantado un sistema de contabilidad de costos para la gestión; por otro, verificar empíricamente la relación existente entre el grado de utilización de sistemas de costos sobre el rendimiento de la Pyme. Para ello se realiza un estudio empírico con 1,425 Pyme del estado de Veracruz (México). Las Pyme con un mayor grado de implantación de modelos de costos se caracterizan por ser empresas medianas, maduras, no familiares y dirigidas por gerentes con formación universitaria. Asimismo, se trata de empresas muy innovadoras, con una tecnología avanzada y que disponen de certificación de calidad. Adicionalmente, los resultados muestran que la implantación de un sistema de costos ejerce una influencia positiva sobre la rentabilidad de la Pyme.

Palabras clave: costos, rentabilidad, Pyme.

\section{Introducción}

耳 $\mathrm{n}$ los últimos años hemos asistido a un creciente interés, tanto en el mundo - académico como en el profesional, en analizar la utilidad de implantar un sistema de contabilidad de costos en la estructura organizativa de la empresa. El control de la gestión se convierte en un elemento básico para la toma de decisiones.

\footnotetext{
* Profesor-investigador de la Universidad Politécnica de Cartagena, España. Correo electrónico: domingo.garcia@upct.es

** Profesor-investigador de la Universidad de Murcia, España. Correo electrónico: salvlau@um.es

$* * *$ Profesor-investigador de la Universidad de Cantabria, España. Correo electrónico: francisco.martinez@unican.es
} 
Por ello, los sistemas de información, si están bien desarrollados y estructurados, pueden considerarse una ventaja competitiva sostenible (Barney, 1991). Los sistemas de contabilidad de costos deberían constituir herramientas comunes en los sistemas organizativos de todas las empresas con independencia de su tamaño. Sin embargo, existen numerosos estudios que ponen de manifiesto que la utilización de sistemas de control de gestión no están lo suficientemente extendidos en las Pyme (Holmes y Nicholls, 1989; Merz y Sauber, 1995; Choe, 1996; Chenhall y LangfieldSmith, 1998). La dirección de la empresa debe basar sus decisiones en función de datos objetivos que sólo se pueden obtener si la empresa utiliza las distintas técnicas económicas que están a su disposición.

Por lo anterior, el objetivo de este trabajo es doble: por una parte, caracterizar a las empresas que utilizan la contabilidad de costos en el seno de sus organizaciones; por otra, verificar empíricamente la relación existente entre el grado de utilización de la contabilidad de costos y el rendimiento de la Pyme. Para ello se llevó a cabo un estudio empírico sobre una muestra de 1,425 Pyme del estado de Veracruz (México). La complejidad y dinamismo del actual entorno empresarial hace necesario un profundo conocimiento de las organizaciones y de cuáles son las variables o factores que pueden considerarse claves para su éxito competitivo. La cuestión fundamental por responder es: ¿la implantación de un sistema de contabilidad de costos puede ayudar al éxito competitivo de la Pyme?

El trabajo se ha estructurado de la siguiente forma: en primer lugar, exponemos un breve marco conceptual sobrelos objetivosy lautilidadde la contabilidad decostos; en segundo, mostramos la metodología del estudio empírico, las características de la muestra y la justificación de las variables utilizadas; en tercero, llevamos a cabo el análisis de los resultados; finalmente, extraemos las principales conclusiones del estudio.

\section{Objetivos y utilidad de la contabilidad de costos}

La contabilidadde $\operatorname{costos}^{1}$ ha ido evolucionando ampliamente en las últimas décadas. Hasta 1950 el objetivo principal era la determinación de los costos y el control

\footnotetext{
${ }^{1}$ Aunque a veces y para efectos prácticos resulta una mera cuestión semántica, señalamos que no existe una clara unanimidad entre los distintos autores en cuanto al término por utilizar. Así, también es usual denominar esta disciplina como: contabilidad de costes, industrial, analítica de la explotación, o el término anglosajón, management o managerial accounting (contabilidad de gestión).
} 
financiero. En las décadas siguientes los esfuerzos se centraron en la elaboración de información para la planificación y el control de la gestión, apareciendo técnicas de análisis de las decisiones y la contabilidad de responsabilidades. Desde 1985 se le añaden los trabajos para la reducción de los "desperdicios" y mermas de los recursos utilizados partiendo del análisis de los procesos y las técnicas de gestión de costos. En la actualidad se prioriza el análisis de los procesos de creación de valor y el uso efectivo de los recursos, a través de instrumentos y técnicas que nos permitan analizar los inductores de valor (drivers) yla innovación organizativa (IFAC, 1998; AECA, 2005).

Dadas las pretensiones de este trabajo, queremos delimitar inicialmente el contenido y objetivos de la contabilidad de costos; para ello realizaremos el siguiente recorrido lógico-deductivo. Para Schneider (1968) la contabilidad interna o industrial se refiere al ciclo técnico de la corriente de riqueza de la empresa, en la cual tiene lugar el proceso de producción mediante la elaboración de las primeras materias. En este proceso de producción, los costos - como expresión de los bienes que se consumen en éste- y la producción — como expresión de los bienes que se obtienen del proceso mencionado- ocupan un lugar central.

Por su parte, Fernández Pirla (1977a) señala que bajo la denominación general de contabilidad de costos se comprende en la literatura contable el estudio de los procedimientos contables y aun estadísticos dirigidos al conocimiento del costo en sus distintos estratos o manifestaciones y en las diferentes empresas. Además, interpreta que el estudio de la magnitud costo está íntimamente relacionado con la investigación y el cálculo de rendimientos. Por consiguiente, la contabilidad de costos no sólo comprende el estudio de los costos en sentido estricto, sino también el de la productividad y rendimientos. Aunque continúa su exposición indicando que el cálculo de los costos y la determinación de la productividad no es un problema estrictamente contable, precisando que el análisis del costo y de la productividad en el orden económico real de la empresa pertenece propiamente a la economía de ésta; también este autor indica que el conocimiento y la investigación de estas magnitudes económicas se verifica fundamentalmente a través de la contabilidad, particularmente en el ámbito de la contabilidad de costos.

En la misma línea, Cañibano Calvo (1979) entiende que el sistema de costos podría ser considerado como el conjunto de elementos que pretenden conseguir el cálculo más idóneo posible del costo de los outputs del sistema de acuerdo con los objetivos de planificación y control, y dentro del más amplio sistema de la empresa en la cual ha de integrarse. Para Dearden (1976), la contabilidad de costos es aquella rama de 
la contabilidad destinada a medir los recursos económicos intercambiados o consumidos (o que vayan a serlo) en la producción de bienes o prestación de servicios. Shillinglaw (1977) define ampliamente la contabilidad de costos como el conjunto de conceptos, métodos y procedimientos usados para medir, analizar o estimar los costos, rentabilidad y rendimientos de los distintos productos, departamentos y otros sectores de las operaciones de una empresa, ya sea para uso interno o externo o ya sea para ambas aplicaciones, con el fin de informar de estas cuestiones a las partes interesadas. De esta forma, en la definición del concepto se percibe la interrelación entre la contabilidad financiera y la analítica.

En cuanto al objeto de la contabilidad de costos, Margerin y Ausset (1982) señalan tres: el cálculo de los costos de los productos o servicios, el control de la gestión y el análisis económico y la toma de decisiones. No obstante, y en relación con este último objeto, "la toma de decisiones" no le correspondería en un sentido teórico-conceptual a la contabilidad de costos porque ésta no viene definida por este objeto, sino que su elaboración es un medio para realizar esa etapa decisional por quien corresponda.

En definitiva, la contabilidad de costos tiene por objeto no sólo reclasificar las cargas o costos de la contabilidad financiera según su destino, sino constituir una base de datos de información económica, interna a la empresa, a la que todo responsable de la empresa puede acceder para obtener la información necesaria con el fin de establecer una adecuada toma de sus decisiones.

La contabilidad de costos es un sistema de información cuyos objetivos principales, de acuerdo con Horngren (1984), son:1) elaborarinformes internos para los responsables de la gestión con el fin de ser empleados tanto en la planificación y control de las operaciones corrientes como en la formulación de planes y políticas más amplias; y 2) valorar los inventarios y determinar la renta, que satisface simultáneamente la demanda de información de terceras personas y de los directivos de la empresa. El plan contable francés (1982), en suapartado de definicióny objetivos de la contabilidad analítica, la define como un modo de tratamiento de datos cuyos objetivos esenciales son: 1) conocer los costos de las diferentes funciones asumidas por la empresa; 2) determinar las bases para la evaluación de ciertos elementos del balance de la empresa; 3) explicar los resultados a través del cálculo de los costos de los productos (bienes y servicios) y su comparación con los precios de venta correspondientes; 4) establecer las previsiones de cargas y de productos corrientes (costos preestablecidos y presupuestos de explotación, por ejemplo); y 5) constatar la realización y explicar las desviaciones que resulten (control de los costos y de los presupuestos, por ejemplo). 
Para la Asociación Española de Contabilidady Administración de Empresas (AECA, 1990), la contabilidad de costos es la que suministra la información analítica relativa a los costos de los productos y servicios generados por la empresa, asumiendo el nivel de desagregación que se considere oportuno en cada situación para determinarel valor de las existencias y el costo de los productos vendidos con el objeto de poder transmitir esta información a los administradores o gerentes con el fin de que se pueda proceder a la confección de los estados contables. Indica, asimismo, que la contabilidad de costos se basa en los hechos históricos y sirve de apoyo a la elaboración de los presupuestos y el cálculo de las magnitudes estándar para, posteriormente, determinar las desviaciones con respecto al mismo. Por otro lado, distingue las siguientes fases del ciclo de la contabilidad de costos: 1) determinación y análisis de los costos de los factores adquiridos; 2) determinación y análisis de los costos incurridos en la realización de las distintas actividades efectuadas en la empresa; y 3) determinación y análisis (composición o estructura) del costo de los productos.

La Asociación Española de Contabilidad Directiva (ACODI, 1992) señala que en realidad los modelos de contabilidad externa y contabilidad interna no pueden considerarse como dos instrumentos diferentes, sino como dos partes o piezas complementarias del mismo instrumento, del mismo modelo, que representan dos subsistemas complementarios del sistema único de la circulación del valor. La información que suministran ambas contabilidades se somete a "procesos contables" para que sea útil a la dirección o gerencia de la Pyme en sus distintos niveles orgánicos, como instrumento de diagnóstico, planeación y control. Esto, pues, es lo que demandan las nuevas tendencias de gestión, los intelectuales, los estudiosos y los buenos gestores de nuestras Pyme; veamos si eso es así en la práctica cotidiana.

Kaplan (1990) y otros (1990-2004) analizan la evolución de la contabilidad de costos hacia la contabilidad de dirección estratégica, indicando que la primera es una herramienta necesaria de la segunda. Así, diversos autores estudian y reclaman una evolución y cambio de la misma para que no sea un mero suministrador de datos, sino que se debe ligar la estrategia con los costos, pues éste es un factor estratégico más. Diversos autores apoyan y relatan esta evolución desde la contabilidad de costos y contabilidad de gestión hacia la contabilidad de dirección estratégica, siendo la contabilidad de costos un vértice necesario de esta pirámide. Desde los años 90 a los actuales, podemos citar a Horngren y Foster (1987), Johansson (1990), Edward y Enmanuel (1990), Innes y Mitchell (1990), Chenhall y Langfield-Smith, (1998), Kennedy (2001), Choe (2004), entre otros. 


\section{Para finalizar este apartado, en las tablas 1 y 2 incluimos diferentes pronunciamientos de organismos profesionales y asociaciones profesionales en relación con el concepto y objetivos de la contabilidad de costos.}

\section{Tabla 1}

\section{Pronunciamientos de organismos profesionales contabilidad de costos/contabilidad de gestión}

\begin{tabular}{|c|c|}
\hline $\begin{array}{l}\text { AMERICAN } \\
\text { ACCOUNTING } \\
\text { ASSOCIATION } \\
\text { (AAA) }\end{array}$ & $\begin{array}{l}\text { Management accounting: supone la aplicación de técnicas y conceptos adecuados para proce- } \\
\text { sar los datos históricos y proyectados de carácter económico de una entidad, con el fin de ayudar } \\
\text { a la dirección a establecer planes para el logro de estos objetivos económicos razonables, así } \\
\text { como para la toma de decisiones racionales tendentes al logro de estos objetivos. Incluye mé- } \\
\text { todos y conceptos necesarios para la planificación efectiva, la elección entre cursos de acción } \\
\text { alternativos y el control a través de la evolución e interpretación de la actuación (AAA-1969). } \\
\text { Objetivos de la contabilidad de gestión: planificación, organización, control directivo, dirección } \\
\text { de sistemas operativos por producto, proyecto u otra segmentación de operaciones (AAA- } \\
\text { 1972) }\end{array}$ \\
\hline $\begin{array}{l}\text { CHARTERED } \\
\text { INSTITUTE OF } \\
\text { MANAGEMENT } \\
\text { ACCOUNTANTS } \\
\text { (CIMA) }\end{array}$ & $\begin{array}{l}\text { La contabilidad de gestión pretende proporcionar información para la gestión, con el propósito } \\
\text { de: formular políticas, planificar y controlar todas las actividades de la empresa, tomar decisio- } \\
\text { nes sobre actuaciones en marcha, proporcionar información a los usuarios externos a la firma, } \\
\text { proporcionar información a los empleados, salvaguardar los activos (CIMA Terminology-1986). } \\
\text { La contabilidad de costos es aquella parte de la contabilidad de gestión encargada de la } \\
\text { confección de presupuestos, establecimiento de costos estándares y reales de las operaciones, } \\
\text { procesos, departamentos o productos, el análisis de las desviaciones y el aprovechamiento y } \\
\text { uso social de los fondos (CIMA Terminology-1986). }\end{array}$ \\
\hline $\begin{array}{l}\text { NATIONAL } \\
\text { ASSOCIATION OF } \\
\text { ACCOUNTANTS } \\
\text { (NAA) O } \\
\text { INSTITUTE OF } \\
\text { MANAGEMENT } \\
\text { ACCOUNTANTS } \\
\text { (IMA) }\end{array}$ & 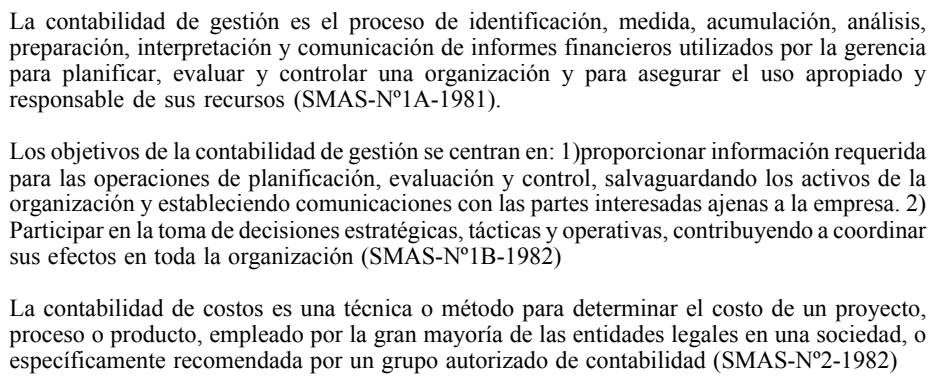 \\
\hline $\begin{array}{l}\text { ASOCIACIÓN } \\
\text { ESPAÑOLA DE } \\
\text { CONTABILIDAD } \\
\text { Y } \\
\text { ADMINISTRACIÓN } \\
\text { DE EMPRESAS } \\
\text { (AECA) }\end{array}$ & $\begin{array}{l}\text { La contabilidad de costos es aquella rama de la contabilidad que suministra la información } \\
\text { analítica relativa a los costos de los productos y servicios generados por la empresa, asumiendo } \\
\text { el nivel de desagregación que se considere oportuno en cada situación, a efectos de determinar } \\
\text { el valor de las existencias y el costo de los productos vendidos, para poder transmitir esta } \\
\text { información a los administradores o gerentes a fin de que puedan proceder a la confección de los } \\
\text { estados contables (AECA- Doc. } \mathrm{N}^{\circ}-1-1989 \text { ) } \\
\text { La contabilidad de gestión es aquella rama de la contabilidad que tiene por objeto la captación, } \\
\text { medida y valoración de la circulación interna, así como su racionalización y control, con el fin } \\
\text { de suministrar a la organización la información relevante para la toma de decisiones empresariales } \\
\text { (AECA-Doc. No 1-1989). } \\
\text { Los objetivos de la contabilidad directiva van ligados a la visión estratégica de la empresa, de } \\
\text { la que se desprenderán decisiones operativas o de gestión, que tratarán de coordinar todo tipo } \\
\text { de esfuerzos en el seno de una organización dentro de un determinado entorno (AECA-Doc. } \\
\mathrm{N}^{\circ} 1-1989 \text { ) }\end{array}$ \\
\hline
\end{tabular}

Fuente: Elaboración propia 
Tabla 2

Objetivos de la contabilidad de costos y de gestión para las asociaciones profesionales contables

\begin{tabular}{|l|c|c|c|c|c|c|}
\hline OBJETIVOS & AECA & ACODI & NAA & IFAC & AAA & CIMA \\
\hline $\begin{array}{l}\text { Suministrar la información } \\
\text { necesaria para la toma de } \\
\text { decisiones }\end{array}$ & $\bullet$ & $\bullet$ & $\bullet$ & $\bullet$ & $\bullet$ & $\bullet$ \\
\hline $\begin{array}{l}\text { Coordinación de las } \\
\text { actividades de la empresa }\end{array}$ & $\bullet$ & - & $\bullet$ & $\bullet$ & $\bullet$ & - \\
\hline Control & $\bullet$ & $\bullet$ & $\bullet$ & $\bullet$ & $\bullet$ & $\bullet$ \\
\hline Motivación & $\bullet$ & - & - & - & $\bullet$ & - \\
\hline $\begin{array}{l}\text { Suministrar información para } \\
\text { las funciones directivas de } \\
\text { planificación y organización }\end{array}$ & $\bullet$ & - & $\bullet$ & $\bullet$ & $\bullet$ & $\bullet$ \\
\hline
\end{tabular}

Fuente: Aibar(2002)

\section{Metodología}

\subsection{Objetivos de la investigación}

En la actualidad ser competitivos, en el sentido de poseer la capacidad de anticipación y respuesta a los retos del entorno, es vital para la supervivencia de las Pyme (Camisón, 1997). Para mejorar este aspecto, la Pyme tiene - entre otros aspectos- la necesidad de adecuar tanto sus estrategias como su estructura organizativa y su forma de gestión al entorno dinámico de la economía actual. En este contexto, la implantación de la contabilidad de costos puede constituir una herramienta de primer orden para la gestión de la Pyme y mejorar su competitividad. Los objetivos que nos planteamos en esta investigación son los siguientes:

- Caracterizar a las Pyme que utilizan con mayor intensidad la contabilidad de costos en el seno de sus organizaciones, considerando variables de contingencia - como el tamaño, el sector, su antigüedad, el control familiar de la propiedad y la formación del gerente - y variables competitivas - como la estrategia, la posición tecnológica y la certificación de la calidad.

- Analizar la relación existente entre rendimiento y grado de utilización de la contabilidad de costos. Si vamos a aconsejar a las Pyme la implantación de la contabilidad de costos para el control de su gestión, debemos 
asegurarnos cuáles son los rendimientos esperados frente a las empresas que adopten una actitud más conservadora o unas prácticas de gestión diferentes. En la literatura existe una abundante evidencia empírica que relaciona el grado de utilización de contabilidad de costos y sistemas de control de gestión con el rendimiento de la Pyme (Bright et al., 1992; Chenhall y Langfield-Smith, 1998; Adler et al., 2000; Kennedy y AffleckGraves, 2001).

\subsection{Diseño y cobertura de la muestra}

La muestra utilizada está compuesta por 1,425 Pyme del estado de Veracruz (México). Los datos proceden de la base de datos del proyecto Análisis estratégico para el desarrollo de la pequeña y mediana empresa, estado de Veracruz, patrocinado por la Universidad Veracruzana.

El diseño general de la muestra se basó en los principios del muestreo estratificado en poblaciones finitas. La población de empresas, para los efectos del diseño muestral, ha sido segmentada de acuerdo con tres criterios: región, actividad y tamaño. La variable tamaño se ha definido en función del número de empleados generándose tres grupos: microempresas (hasta 10 trabajadores), empresas pequeñas (de 11 a 50 trabajadores) y empresas medianas (más de 50 trabajadores); se ha excluido de la población el conjunto de empresas con 5 o menos trabajadores. El número de empresas en cada uno de los estratos se ha obtenido de la página web de la Secretaría de Economía del Gobierno Federal de México.

El tamaño muestral fue determinado para lograr que el margen de error máximo para la estimación de una proporción (frecuencia relativa de respuesta en un ítem específico de una cuestión) del conjunto de la muestra fuese inferior a 0.02 puntos con un nivel de confianza del 95\%.

La técnica para recopilar la información fue una encuesta personal realizada por personal calificado del proyecto en la sede de la Pyme; se utilizó como soporte un cuestionario autoadministrado dirigido al gerente de la empresa (en el anexo I se presenta el cuestionario). Se realizaron pruebas de control en el proceso de elaboración de la encuesta. El trabajo de campo se inició el 1 de octubre 2002 y finalizó el 31 de marzo de 2003. Debemos señalar que en el desarrollo de las distintas fases de esta investigación se ha respetado escrupulosamente el secreto estadístico 
de la información utilizada. En la siguiente tabla 3 mostramos la distribución de la muestra y su grado de cobertura.

Tabla 3

Distribución de la población de empresas y de la muestra

\begin{tabular}{|l|r|r|r|}
\hline & muestra & población & cobertura \\
\hline Manufacturas & 328 & 411 & $79,81 \%$ \\
\hline Construcción & 116 & 213 & $54,46 \%$ \\
\hline Transporte & 47 & 90 & $52,22 \%$ \\
\hline $\begin{array}{l}\text { Comercios y } \\
\text { Servicios }\end{array}$ & 867 & 1.589 & $54,56 \%$ \\
\hline Turismo & 67 & 136 & $49,26 \%$ \\
\hline Total & $\mathbf{1 . 4 2 5}$ & $\mathbf{2 . 4 3 9}$ & $\mathbf{5 8 , 4 3 \%}$ \\
\hline
\end{tabular}

\subsection{Medición de las variables}

En este subapartado expondremos de forma sintética la medición de las variables, así como determinadas observaciones para clarificar su elección. Las variables utilizadas son:

\begin{tabular}{|l|l|}
\hline $\begin{array}{l}\text { Grado de utilización de } \\
\text { sistemas de contabilidad } \\
\text { de costos }\end{array}$ & $\begin{array}{l}\text { Se ha medido de forma subjetiva a partir de la opinión del gerente de la } \\
\text { empresa, utilizando una escala tipo Likert de 5 puntos donde 1=mínimo } \\
\text { uso, 5=máximo uso. }\end{array}$ \\
\hline Antigüedad & $\begin{array}{l}\text { Variable continua. Número de años desde la constitución de la empresa. } \\
\text { A partir de esta variable se construye una dicotomía, que toma valor 0 } \\
\text { cuando la empresa tiene menos de 10 años de antigüedad y se denomina } \\
\text { joven; toma valor 1 cuando la empresa tiene 10 o más años y se denomina } \\
\text { madura. Esta recodificación ha sido utilizada previamente por los } \\
\text { estudios de Holmes y Nichols (1989), AECA (2002 y 2005) y Yasuda } \\
(2005) \text {. }\end{array}$ \\
\hline Sector & $\begin{array}{l}\text { Variable nominal distinguiendo 5 categorías: manufacturas, comercios y } \\
\text { servicios, construcción, turismo y transporte. Esta categorización parte } \\
\text { del Sistema de Clasificación Industrial de América del Norte (SCIAN, } \\
\text { 2002). }\end{array}$ \\
\hline
\end{tabular}




\begin{tabular}{|c|c|}
\hline $\begin{array}{l}\text { Estructura de la } \\
\text { propiedad }\end{array}$ & $\begin{array}{l}\text { Se considera empresa familiar aquella que reúne las siguientes } \\
\text { caracteristicas: propiedad y control de la empresa en la misma familia, } \\
\text { influencia de la familia en la toma de decisiones y propósito de transmitir } \\
\text { la empresa a la siguiente generación. (Romano et al., 2000). } \\
\text { Dos variables dicotómicas: control mayoritario familiar (un grupo } \\
\text { familiar tiene más del } 50 \% \text { del capital) o no y puestos de dirección } \\
\text { ocupados por miembros de la familia o no. }\end{array}$ \\
\hline Formación del gerente & $\begin{array}{l}\text { Variable dicotómica: toma valor } 0 \text { cuando el gerente dispone de estudios } \\
\text { primarios, bachiller o equivalente; toma valor } 1 \text { cuando el gerente dispone } \\
\text { de estudios universitarios. }\end{array}$ \\
\hline Tamaño & $\begin{array}{l}\text { Variable continua y nominal. Dos variables (1) número medio de } \\
\text { empleados en } 2002 \text {. A partir de estas variables se construye una nominal, } \\
\text { que toma valor } 0 \text { cuando la empresa es micro (menos de } 10 \text { trabajadores), } \\
\text { valor } 1 \text { cuando la empresa es pequeña ( } 10 \text { a } 50 \text { trabajadores) y toma valor } \\
2 \text { cuando la empresa es mediama (empresa de más de } 50 \text { trabajadores } \\
\text { hasta } 250 \text { ). El estudio no recoge empresas con menos de } 5 \text { empleados. }\end{array}$ \\
\hline Estrategia competitiva & $\begin{array}{l}\text { La clasificación de los tipos de estrategia más utilizada en los estudios } \\
\text { empiricos es la propuesta por Miles y Snow ( } 1978 \text { ) y también la de Porter } \\
\text { (1980). Estas clasificaciones identifican la esencia de la mayor parte de } \\
\text { las posiciones competitivas de la empresa (Kotabe y Duhan. 1993). En } \\
\text { nuestro trabajo utilizaremos la tipologia de Miles y Snow (1978), variable } \\
\text { nominal, donde se distinguen cuatro categorias: estrategia exploradora, } \\
\text { analizadora, defensiva y reactiva. }\end{array}$ \\
\hline Posición tecnológica & $\begin{array}{l}\text { Variable nominal de cuatro categorias: } \\
\text { - Fuerte, la empresa realiza un desarrollo interno de la tecnologia que } \\
\text { utiliza con el fin de obtener mejores resultados que la competencia. } \\
\text { - Buena, la tecnologia adquirida por la empresa o el uso que se hace de } \\
\text { ella la posiciona por delante de la competencia. } \\
\text { - Sostenible, la tecnología que utilizan es la misma que se utiliza en la } \\
\text { mayoria de las empresas del sector y sólo realizan nuevas inversiones } \\
\text { cuando comprueban que la competencia obtiene buenos resultados. } \\
\text { - Débil, la empresa considera que sus principales competidores tienen } \\
\text { una tecnologia más eficiente o moderna que la nuestra. }\end{array}$ \\
\hline Certificación de calidad & $\begin{array}{l}\text { Variable nominal de tres categorías: } \\
\text { - Si, dispone de certificación de calidad. } \\
\text { - No, pero están en proceso previo. } \\
\text { - No, y no están en proceso previo. }\end{array}$ \\
\hline Rentabilidad & $\begin{array}{l}\text { Utilidad neta sobre ventas de la empresa en los dos últimos años. Variable } \\
\text { nominal de cinco categorias: } \\
\text { - I: menos del } 0 \% \text {. } \\
\text { - } 2: \text { del } 0 \% \text { al } 5 \% . \\
\text { - } 3: \text { del } 5 \% \text { al } 10 \% . \\
\text { - } 4: \text { del } 10 \% \text { al } 15 \% \text {. } \\
\text { - } 5 \text { : más del } 15 \% .\end{array}$ \\
\hline
\end{tabular}




\section{Resultados}

\subsection{Características de las Pyme que implantan contabilidad de costos}

En general, se aprecia un escaso grado de implantación de la contabilidad de costos en la estructura organizativa de las Pyme (véase tabla 4). Tan sólo el 19.2\% de las empresas tienen un elevado nivel de desarrollo de la contabilidad de costos o de un sistema de contabilidad de costos. De forma contraria, los resultados muestran que en el $24 \%$ de las Pyme no existe un modelo de contabilidad de costos implantado.

\section{Tabla 4}

\section{Implantación y control de un sistema de contabilidad de costos $\%$ total de empresas}

\begin{tabular}{|cc|ccc|}
\hline Nulo & Poco & Medio & Alto & Máximo \\
$24,0 \%$ & $12,3 \%$ & $25,2 \%$ & $19,3 \%$ & $19,2 \%$ \\
\hline
\end{tabular}

Si analizamos con más detalle las características de las Pyme, en función de diferentes factores, podemos encontrar las siguientes diferencias significativas:

- Tamaño: las empresas medianas son las que utilizan en mayor medida la contabilidad de costos (ver tabla 5). En el 38.1\% de las empresas medianas se da un grado de desarrollo máximo. Estos porcentajes bajan al $24.3 \%$ en las empresas pequeñas y al $12.2 \%$ en las microempresas.

- Sector: las empresas de transportes son más proclives a implantar sistemas de contabilidad de costos (tabla 6). En el $33.3 \%$ de los casos existe un máximo grado de implantación. Contrariamente, en las empresas de manufactura (debido sobre todo por su reducido tamaño) encontramos que sólo el 13.3\% lo implanta con un elevado grado de desarrollo.

- Antigüedad: las empresas de mayor edad, 23\% de los casos, tienen extendido en su organización con mayor profundidad sistemas de contabilidad de costos que las empresas jóvenes, $16.7 \%$ de los casos (tabla 7).

- Formación del gerente: las empresas con gerentes universitarios disponen en mayor proporción sistemas de contabilidad de costos, $22.5 \%$, que las empresas con gerentes no universitarios, $15.3 \%$ (tabla 8).

- Estructura de la propiedad: las empresas no familiares tienen un mayor grado de utilización que las empresas familiares (tabla 9). E1 21.3\% de las 
empresas no familiares tienen un grado de implantación muy alto de sistemas de contabilidad de costos, frente al $18.7 \%$ de las empresas familiares.

- Estrategia: las empresas con una actitud más innovadora para competir en los mercados adoptan con mayor intensidad sistemas de contabilidad de costos (tabla 10). Así, el 32.7\% de las empresas exploradoras tienen un grado de utilización máximo, frente al 19.3\% de las empresas analizadoras, al 14.3\% de las defensivas o al $8.3 \%$ de las empresas que tienen una estrategia reactiva.

- Posición tecnológica: las empresas con un nivel tecnológico más avanzado hacen un uso más extensivo de sistemas de contabilidad de costos (tabla 11). De esta forma, el 36\% de las empresas con una tecnología fuerte tienen muy desarrollado la contabilidad de costos en su organización, frente al $20.6 \%$ de las empresas con una tecnología buena, al $12.1 \%$ con tecnología sostenible o al 9.3\% con una tecnología débil.

- Calidad: las empresas que disponen de certificación de calidad, o están en proceso para obtenerla, tienen implantados en mayor proporción sistemas de contabilidad de costos más desarrollados (tabla 12). Así, el 31.4\% de las empresas con certificación de calidad y el $24.4 \%$ de las que están en proceso, tiene un grado máximo de implantación de sistemas de contabilidad de costos, frente al 15.9\% de las empresas que no disponen de certificación de calidad.

\section{Tabla 5}

\section{Sistema de contabilidad de costos por tamaño}

Valoración del ítem: 1: Nula implantación a 5: Máxima implantación Anova de un factor, sig. F: 0,000 
Tabla 6

Sistema de contabilidad de costos por sector

\begin{tabular}{|l|c|c|c|c|}
\hline & Media & Desv. Típica & $\begin{array}{c}\% \text { empresas } \\
\text { implantación } \\
(1) \text { Nula }\end{array}$ & $\begin{array}{c}\text { \% empresas } \\
\text { implantación } \\
(5) \text { Máxima }\end{array}$ \\
\hline Manufacturas & 2,59 & 1,406 & $31,8 \%$ & $13,3 \%$ \\
\hline $\begin{array}{l}\text { Comercios y } \\
\text { Servicios }\end{array}$ & 3,06 & 1,413 & $21,9 \%$ & $20,1 \%$ \\
\hline Construcción & 3,10 & 1,476 & $24,8 \%$ & $23,0 \%$ \\
\hline Turismo & 3,21 & 1,309 & $14,9 \%$ & $20,9 \%$ \\
\hline Transporte & 3,33 & 1,604 & $22,2 \%$ & $33,3 \%$ \\
\hline
\end{tabular}

Valoración del ítem: 1: Nula implantación a 5: Máxima implantación Anova de un factor, sig. F: 0,000

Tabla 7

Sistema de contabilidad de costos por antigüedad

\begin{tabular}{|l|c|c|c|c|}
\hline & Media & Desv. Típica & $\begin{array}{c}\text { \% empresas } \\
\text { implantación } \\
\text { (1) Nula }\end{array}$ & $\begin{array}{c}\text { \% empresas } \\
\text { implantación } \\
\text { (5) Máxima }\end{array}$ \\
\hline Jóvenes & 2,87 & 1,417 & $26,1 \%$ & $16,7 \%$ \\
\hline Maduras & 3,14 & 1,435 & $20,4 \%$ & $23,0 \%$ \\
\hline
\end{tabular}

Valoración del ítem: 1: Nula implantación a 5: Máxima implantación Anova de un factor, sig. F: 0,001

Tabla 8

Sistema de contabilidad de costos por nivel de formación del gerente

\begin{tabular}{|l|c|c|c|c|}
\hline & Media & Desv. Típica & $\begin{array}{c}\text { \% empresas } \\
\text { implantación } \\
(1) \text { Nula }\end{array}$ & $\begin{array}{c}\% \text { empresas } \\
\text { implantación } \\
\text { (5) Máxima }\end{array}$ \\
\hline No Universitarios & 2,62 & 1,465 & $34,8 \%$ & $15,3 \%$ \\
\hline Universitarios & 3,24 & 1,356 & $16,3 \%$ & $22,5 \%$ \\
\hline
\end{tabular}

Valoración del ítem: 1: Nula implantación a 5: Máxima implantación Anova de un factor, sig. F: 0,000 
Tabla9

Sistema de contabilidad de costos por estructura de la propiedad

\begin{tabular}{|l|c|c|c|c|}
\hline & Media & Desv. Típica & $\begin{array}{c}\text { \% empresas } \\
\text { implantación } \\
\text { (1) Nula }\end{array}$ & $\begin{array}{c}\text { \% empresas } \\
\text { implantación } \\
\text { (5) Máxima }\end{array}$ \\
\hline Empresa familiar & 2,93 & 1,439 & $25,4 \%$ & $18,7 \%$ \\
\hline Empresa no familiar & 3,16 & 1,386 & $18,9 \%$ & $21,3 \%$ \\
\hline
\end{tabular}

Valoración del ítem: 1: Nula implantación a 5: Máxima implantación Anova de un factor, sig. F: 0,014

Tabla 10

Sistema de contabilidad de costos por estrategia competitiva

\begin{tabular}{|l|c|c|c|c|}
\hline & Media & Desv. Típica & $\begin{array}{c}\text { \% empresas } \\
\text { implantación } \\
(1) \text { Nula }\end{array}$ & $\begin{array}{c}\text { \% empresas } \\
\text { implantación } \\
(5) \text { Máxima }\end{array}$ \\
\hline Exploradora & 3,56 & 1,342 & $12,1 \%$ & $32,7 \%$ \\
\hline Analizadora & 3,16 & 1,336 & $17,0 \%$ & $19,3 \%$ \\
\hline Defensiva & 2,71 & 1,408 & $30,1 \%$ & $14,3 \%$ \\
\hline Reactiva & 2,23 & 1,348 & $44,2 \%$ & $8,3 \%$ \\
\hline
\end{tabular}

Valoración del ítem: 1: Nula implantación a 5: Máxima implantación Anova de un factor, sig. F: 0,000

Tabla 11

\section{Sistema de contabilidad de costos por posición tecnológica}

\begin{tabular}{|l|c|c|c|c|}
\hline & Media & Desv. Típica & $\begin{array}{c}\text { \% empresas } \\
\text { implantación } \\
\text { (1) Nula }\end{array}$ & $\begin{array}{c}\text { \% empresas } \\
\text { implantación } \\
\text { (5) Máxima }\end{array}$ \\
\hline Fuerte & 3,56 & 1,414 & $14,3 \%$ & $36,0 \%$ \\
\hline Buena & 3,26 & 1,301 & $15,3 \%$ & $20,6 \%$ \\
\hline Sostenible & 2,63 & 1,382 & $30,6 \%$ & $12,1 \%$ \\
\hline Débil & 2,21 & 1,401 & $49,2 \%$ & $9,3 \%$ \\
\hline
\end{tabular}

Valoración del ítem: 1: Nula implantación a 5: Máxima implantación Anova de un factor, sig. F: 0,000 
Tabla 12

Sistema de contabilidad de costos por certificación de calidad

\begin{tabular}{|l|c|c|c|c|}
\hline & Media & Desv. Típica & $\begin{array}{c}\text { \% empresas } \\
\text { implantación } \\
(1) \text { Nula }\end{array}$ & $\begin{array}{c}\text { \% empresas } \\
\text { implantación } \\
\text { (5) Máxima }\end{array}$ \\
\hline Sí dispone & 3,38 & 1,483 & $18,3 \%$ & $31,7 \%$ \\
\hline En proceso & 3,35 & 1,335 & $14,0 \%$ & $24,4 \%$ \\
\hline No dispone & 2,80 & 1,421 & $28,0 \%$ & $15,9 \%$ \\
\hline
\end{tabular}

Valoración delítem: 1: Nula implantación a 5: Máxima implantación Anova de un factor, sig. F: 0,000

\subsection{Rendimiento y sistemas de contabilidad de costos}

Para verificar la relación existente entre el rendimiento de la Pyme y el grado de utilización de sistemas de contabilidad de costos se plantea el siguiente modelo:

$\begin{array}{ll}\text { Rto }_{\mathrm{i}}=\mathrm{b}_{0}+\mathrm{b}_{1} \text { Tamaño }_{\mathrm{i}}+\mathrm{b}_{2} \text { Edad }_{\mathrm{i}}+\mathrm{b}_{3} \operatorname{Costos}_{\mathrm{i}}+\varepsilon_{\mathrm{i}} \\ \begin{array}{l}\text { Dependiente } \\ \text { Rtoi }\end{array} & \text { Utilidad neta sobre ventas } \\ \text { Control } & \text { Número de empleados } \\ \text { Tamaño } & \text { Valor 0: empresa de 10 o menos años y } \\ \text { Edad } & \text { Valor 1: empresa más de 10 años } \\ & \\ \text { Explicativa } & \text { Grado de utilización de la contabilidad de costos } \\ \text { Costos } & \end{array}$

Para estudiar el efecto del grado de utilización de la implantación de un sistema de contabilidad de costos sobre el rendimiento de la Pyme, se debe analizar el coeficiente $b_{3}$ de la variable costos y su valor t-student asociado para saber si esta influencia es significativa. La tabla 13 muestra los resultados de la regresión por MCO; ahí podemos apreciar que el coeficiente de $b_{3}(0.202)$ es positivo y 
significativo al 99\%. Eso implica que a mayor grado de utilización de sistemas de contabilidad de costos, mayor es la utilizad neta que obtiene la Pyme; por lo tanto, se verifica la hipótesis de trabajo planteada. Esta relación positiva confirma los resultados empíricos previos. Sin embargo, los coeficientes de las variables de control (tamaño y edad) no resultan significativos.

Tabla13

Impacto de la contabilidad de costos sobre la rentabilidad de la Pyme

\begin{tabular}{|c|c|c|c|}
\hline & & Beta & FIV \\
\hline \multirow{4}{*}{ Rentabilidad } & Tamaño & $\begin{array}{l}0.039 \\
(1.379)\end{array}$ & 1.012 \\
\hline & Edad & $\begin{array}{l}0.017 \\
(0.583)\end{array}$ & 1.013 \\
\hline & COSTOS & $\begin{array}{l}0.202 * * * \\
(7.114)\end{array}$ & 1.018 \\
\hline & \begin{tabular}{|l}
$\mathrm{F}$ \\
$\mathrm{R}^{2}$ ajustado
\end{tabular} & $\begin{array}{l}18.855^{* * *} \\
0.043\end{array}$ & \\
\hline
\end{tabular}

$(* * *): \mathrm{p}<0,01$

Beta: coeficientes estandarizados (entre paréntesis t value)

FIV: Factor de Inflación de la Varianza de los parámetros

La significación conjunta del modelo es aceptada, dado que el valor de la $\mathrm{F}$ es significativo al $99 \%$ y un $\mathrm{R}^{2}$ ajustado del $4.3 \%$. También comprobamos que los regresores incluidos en el modelo presentan un factor de inflación de la varianza (VIF) cercano a la unidad, por lo que descartamos la presencia de multicolinealidad.

\section{Conclusiones}

Las Pyme necesitan, cada vez más, establecer mecanismos de control que ayuden a la gerencia a tomar correctamente sus decisiones. La fuerte competencia que en la actualidad provoca, entre otros más cercanos, la globalización de los mercados y el cambiotecnológico está motivando a las Pyme a desarrollar en el seno de su organización sistemas de control de gestión (AECA, 2005). La contabilidad de costos permite a la dirección de la empresa elaborar información para la toma de decisiones respecto de la valoración de los inventarios, del control de costos, la medición de los ingresos-costosbeneficios y analizar la rentabilidad de los productos y mercados. 
En el pasado, la contabilidad de costos se utilizaba exclusiva y fundamentalmente para el control de costos y el análisis de los resultados de la gestión. Sin embargo, el desarrollo de nuevas técnicas de costos han logrado variar los objetivos de ésta, los cuales en la actualidad son: a) la mejora de la rentabilidad del producto, b) la reducción de costos y c) la obtención de una información más ágil y relevante para la gestión. (Bright et al., 1992). En este sentido, la implantación de un sistema de contabilidad de costos en la Pyme puede constituir, si está bien orientado, una ventaja competitiva.

En este trabajo se ha analizado, por una parte, las características de las Pyme que tienen implantado un sistema de contabilidad de costos en su organizacióny, por otra, la relación existente entre el grado de utilización de sistemas de contabilidad de costos y el rendimiento de la Pyme, utilizando una muestra de 1,425 Pyme del estado de Veracruz (México).

Las Pyme que tienen implantado un elevado desarrollo de sistemas de contabilidad de costos se caracterizan por ser empresas medianas y maduras, así como por ser empresas no familiares y gestionadas por gerentes con formación universitaria. Asimismo, si consideramos factores estratégicos, podemos comprobar que las empresas más innovadoras que siguen una estrategia exploradora, las que tienen un gran nivel tecnológico y las que disponen de certificación de calidad, tienen implantado un mayor grado de desarrollo en sus sistemas de costos.

Adicionalmente, el estudio empírico provee información acerca del impacto positivo del grado de utilización de los sistemas de contabilidad de costos sobre el rendimiento de la Pyme. Se confirma, por lo tanto, que su implantación en la estructura organizativa de la empresa puede ser un factor relevante para la mejora de la competitividad de la Pyme.

\section{Bibliografía}

ACODI (1992): Contabilidad directiva, documento No 1, Asociación Española de Contabilidad Directiva, Madrid.

AECA (1990): El marco de la contabilidad de gestión, documento $\mathrm{N}^{\circ} 1$ de la Serie Principios de Contabilidad de Gestión, Asociación Española de Contabilidad y Administración de Empresas, Madrid. 
AECA (2002): Factores determinantes de la eficiencia y rentabilidad de las Pyme en España, Estudios Empíricos, Asociación Española de Contabilidad y Administración de Empresas, Madrid.

AECA (2005): Estrategia e innovación de la Pyme industrial en España, Estudios Empíricos. Asociación Española de Contabilidad y Administración de Empresas, Madrid.

ADLER, R., Everett, A.M. y Waldron, M. (2000): “Advanced management accounting techinques in manufacturing: utilization, benefits, and barriers to implementation", Accounting Forum, 24(2), junio, pp. 131-150.

AIBAR GUZMÁN, B. (2002): Proyecto docente, Universidad de Santiago de Compostela, Santiago de Compostela.

BARNEY, J.B. (1991): "Firm resources and sustained competitive advantage", Journal of Management, Vol. 17, $\mathrm{n}^{\circ}$ 1, pp. 99-120.

BRIGHT, J. et al.,(1992): "The deployment of costing techniques and practices: a UK study", Management Accounting Research, 3, pp. 201-211.

CAMISÓN, C. (1996): "Competitividad y teoría de la estrategia: un análisis aplicado a la cohabitación pyme-gran empresa", Revista Asturiana de Economía, $\mathrm{n}^{\mathrm{o}} 6$, pp. 63-101.

(1997): La competitividad de la Pyme industrial española: estrategia y competencias distintivas, Civitas, Madrid.

CAÑIBANO CALVO, L. (1979): Teoría actual de la contabilidad, ICE, Madrid.

COOPER, R. y R. S. Kaplan, (1991): The Desing of Cost Management Systems, Prentice-Hall International, EEUU.

CHENHALL, R.H. y K. Langfield-Smith, (1998): “The relationship between strategic priorities, management techniques and management accounting: an empirical investigation using a systems approach", Accounting, Organizations and Society, 23(3), pp. 243-264. 
CHOE, J.-M. (1996): “The relationships among performance of accounting information systems, influence factors, and evolution level of information systems", Journal of Management Information Systems, 12(4), pp. 215-239.

(2004): "The relationships among management accounting information, organizational learning and production performance", Journal of Strategic Information Systems, 13, pp. 61-85.

DEARDEN, J. (1976): Sistemas de contabilidad de costos y de control financiero, Deusto, Bilbao.

EDWARD, E.O. y C.R. Emmanuel, (1990): "Diverging view no the boundaries of management accounting", Management Accounting Review, marzo.

FERNÁNDEZ PIRLA, J.M. (1983): Teoría económica de la contabilidad, 10a. edición, ICE, Madrid.

GARCÍA PÉREZ DE LEMA, D. (dir.) (2004): Análisis estratégico para el desarrollo de la pequeña y mediana empresa (estado de Veracruz), Instituto Mexicano de Contadores Públicos, A.C. y Servicio de Publicaciones de la Universidad de Cantabria. México.

HOLMES, S. y D. Nicholls, (1989): "Modelling the Accounting Information Requirements of Small Business", Accounting and Business Research, 19(74), pp. 143-150.

HORNGREN, C.T. (1984): Introduction to management accounting, PrenticeHall, New Jersey.

y G. Foster, (1987): Cost Accounting: a managerial emphasis, PrenticeHall International, New Jersey.

INNES, J. y F. Mitchell, (1990): "The process of change in management accounting: some field study evidence", Management Accounting Research, marzo.

INTERNATIONAL FEDERATION OF ACCOUNTANTS-IFAC (1989): Proposed International Management Accounting Concepts, Exposure Draft 1, abril. 
(1998): International Management Accounting Practice, Statement $\mathrm{N}^{\mathrm{o}}$

1. Management Accounting Concept, Financial and Management Accounting Committee, marzo.

KAPLAN, R.S. (1990): “Diseño de un sistema de costes", Partida doble, octubre.

KENNEDY, T. y J. Affleck-Grave, (2001): "The impact of Activity-Based Costing Techniques on Firm Performance", Journal of Management Accounting Research, 13, pp. 19-45.

MARGERIN, J. y G. Ausset, (1982): Comptabilité analytique; Outil de gestion aide a la dècision, 4a. edición, Les 1 Editions d'Organisation, París.

MERZ, G.R. y Sauber, M.H. (1995): "Profiles of managerial activities in small firms", Strategic Management Journal, 16, pp. 551-564.

MILES, R.E. y Snow, C.C. (1978): Organizational strategy, structure and process, McGraw-Hill, Nueva York.

ROMANO, C.A., G.A. Tanewski y K.X. Smyrnios, (2000): "Capital structure decision making: a model for family business", Journal of Business Venturing, 16, pp. 285-310.

SCHNEIDER, E.(1968): Contabilidad industrial, Aguilar, Madrid.

SHILLINGLAW, G.(1977): Contabilidad de costos. Análisis y control, El Ateneo, Buenos Aires.

YASUDA, T. (2005): "Firm Growth, Size, Age and Behavior in Japanese Manufacturing", Small Business Economics, 24, pp. 1-15. G 


\section{ANEXO I \\ EL CUESTIONARIO}

¿Cuántos años lleva funcionando su empresa?............

años

¿El control mayoritario de su empresa es familiar? (Un grupo familiar tiene más del $50 \%$ del capital):

$\square$ SÍ $\square$ NO

¿Cuál es el nivel de formación reglada del director general / gerente / propietario?:

(Marque sólo una respuesta)

Estudios primarios

Educación secundaria

Bachillerato / preparatoria

Estudios universitarios

A continuación se le presentan cuatro tipos de empresas. Por favor, indique con cuál de ellas se identifica la suya atendiendo a su actuación en los dos últimos años (MARQUE SÓLO UNA RESPUESTA):

Tipo A.- Suele realizar cambios y mejoras en los productos / servicios y mercados con relativa frecuencia, tratando de ser la primera en desarrollar nuevos productos / servicios, aun con el riesgo de que estas innovaciones no tengan éxito.

Tipo B.- Mantiene una base relativamente estable de productos / servicios y mercados, mientras que al mismo tiempo desarrolla de forma selectiva nuevos productos / servicios y mercados, tratando de imitar a las empresas que ya los desarrollaron y tuvieron éxito.

$\underline{\text { Tipo C.- }}$ - Ofrece un conjunto relativamente estable de productos / servicios para un mercado relativamente estable. No está interesada en las modificaciones sino que se concentra en la mejora continua del trabajo dentro de su campo de actuación.

Tipo D.- No cuenta con un área de producto-mercado duradera y estable. Normalmente actúa forzada por las presiones del entorno y de la competencia.

La posición tecnológica de su empresa se puede considerar (MARQUE SÓLO UNA RESPUESTA):

\begin{tabular}{|l|l|}
\hline$\square$ FUERTE & $\begin{array}{l}\text { Desarrollo interno de la tecnología que utilizamos con el fin de obtener mejores } \\
\text { resultados que la competencia. }\end{array}$ \\
\hline$\square$ BUENA & $\begin{array}{l}\text { La tecnología adquirida por la empresa o el uso que se hace de ella nos posiciona } \\
\text { por delante de la competencia. }\end{array}$ \\
\hline$\square$ SOSTENIBLEE & $\begin{array}{l}\text { La tecnología que utilizamos es la misma que se utiliza en la mayoría de empresas } \\
\text { del sector y sólo realizamos nuevas inversiones cuando comprobamos que la } \\
\text { competencia obtiene buenos resultados. }\end{array}$ \\
\hline$\square$ DÉBIL & $\begin{array}{l}\text { Nuestros principales competidores tienen una tecnología más eficiente o moderna } \\
\text { que la nuestra. }\end{array}$ \\
\hline
\end{tabular}

¿Dispone su empresa de una certificación ISO de la serie 9000 o equivalentes?

SI $\quad \square \quad$ Indique la norma y el organismo que lo certificó

NO $\quad \square \quad$ Pero estamos en el proceso previo para la certificación de la calidad

NO $\quad \square \quad$ Y no estamos en el proceso previo para la certificación de la calidad

¿Cuál fue la utilidad neta sobre ventas de su empresa en los dos últimos años?

\begin{tabular}{|c|c|c|c|c|}
\hline Menos del $0 \%$ & Del $0 \%$ al $5 \%$ & Del $5 \%$ al $10 \%$ & Del $10 \%$ al $15 \%$ & Más del $15 \%$ \\
\hline$\square$ & $\square$ & $\square$ & $\square$ & $\square$ \\
\hline
\end{tabular}

